

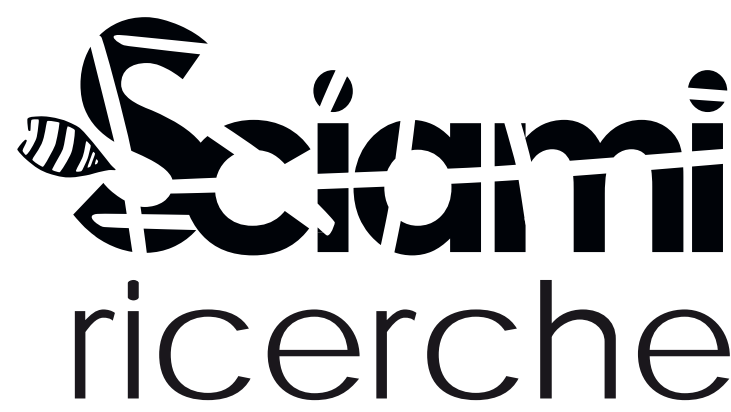

Webzine di Teatro, Video e Suono

Diretta da Valentina Valentini 


\section{COMITATO SCIENTIFICO}

Roberto Calabretto, Università degli Studi di Udine, Federica Dal Falco, Sapienza Università di Roma Francesco Fiorentino, Università degli Studi Roma Tre, Helga Finter, Università di Giessen, Germania, Cristina Grazioli, Università degli Studi d Padova, Carlo Martino, Sapienza Università di Roma, Roberto Pinto, Università degl Studi di Bologna, Cosetta G. Saba, Università degli Studi di Udine, Annalisa Sacchi, Università IUAV di Venezia, Valentina Valentini, Sapienza Università di Roma.

\section{COMITATO EDITORIALE}

Guido Bartorelli, Università degli Studi di Padova, Donata Chiricò, Università della Calabria, Massimo Fusillo, Università degli Studi dell'Aquila, Francesca Gallo Sapienza Università di Roma, Giovanni Iorio Giannoli, già Università degli Studi di Roma Tor Vergata, Thomas Haskell Simpson, Northwestern Università di Chicago, USA, Didier Plassard, Università Paul Valery di Montpellier, Francia, Emanuele Senici, Sapienza Università di Roma, Carlo Serra, Università della Calabria, Francesco Spampinato, Università degli Studi di Bologna.

\section{REDAZIONE}

Samuele Briatore, Dalila D'Amico, Lisa Parolo, Mauro Petruzziello, Stefano Scipioni, Andrea Vecchia, Daniele Vergni.

La webzine aderisce alle linee guida etiche indicate dal COPE Code of Conduct for Journal Editors ${ }^{1}$. Tutti gli articoli vengono esaminati da revisori esterni. Gli articol richiesti e concordati dalla direzione della rivista, secondo il programma editoriale, vengono sottoposti alla valutazione dei membri del comitato scientifico; questa circostanza è segnalata in nota, nella prima pagina del contributo. Sono ammess direttamente dal comitato editoriale i contributi non rilevanti per le finalità che presiedono alla classificazione delle Riviste (schede bibliografiche, forum, interviste, interventi di artisti).

1 publicationethics.org/files/Code_of_conduct_for_journal_editors_Mar11.pdf
Copertina

Eugenio Carmi, frame tratto dal video Olivo verde vivo, 1977, disegno intarsiato con immagini di repertorio, Archivio Eugenio Carmi.

\section{Retro di copertina}

Altair4 Multimedia, scenografia virtuale per MediaMente, 1999, Interconnessioni, Archivio Altair4 Multimedia.

I materiali utilizzati all'interno della pubblicazione (testo, foto, grafiche, etc...) e sulla webzine sono di proprietà dei rispettivi licenziatari e sono stati gentilmente autorizzati solo ed esclusivamente per questa pubblicazione. Per qualunque altro utilizzo è necessario informare i titolari. Per tutte le opere riprodotte, l'editore ha effettuato, senza successo, tutte le ricerche necessarie al fine di identificare gli aventi titolo. Pertanto resta disponibile ad assolvere le proprie obbligazioni.

(C) 2020 - Sciami edizioni (Teramo - Roma)

ISSN: 2532-3830

DOI https://doi.org/10.47109/010220

Registrato presso il ROC al n. 26708

Registrato presso il Tribunale di Roma al n. 169/2018

Sciami|ricerche, n. 8, ottobre 2020

Webzine: https://webzine.sciami.com/webzine/numero-8

www.sciami.com / webzine.sciami.com

Redazione e direzione: via del Mandrione, 451 - 00181 Roma (RM)

Editore: Sciami| edizioni, Largo San Carlo, 9 - 64100 Teramo (TE)

Telefono +390861 247885

E-mailinfo@sciami.com 


\section{US:

|IIIIIIIIIIIIIIIIIIIIIIIII

\section{GLI ARTISTI VISIVI E I LINGUAGGI DELLA TELEVISIONE IN ITALIA: COLLABORAZIONI, SPERIMENTAZIONI E INCURSIONI}

Francesca Gallo, Paola Lagonigro, Martina Rossi

1 Editoriale

Francesca Gallo

4 Integrazione VS decostruzione nelle collaborazioni degli artisti visivi con la tv e qualche nota su Foligno '67 di Alfredo Di Laura

Silvia Bordini

16 La tv come casa dell'arte. Sperimentazioni e utopie Chiara Mari

24 I "Concetti spaziali per televisione" di Lucio Fontana: dalle proposte del Manifiesto Blanco ai primi ambienti Guido Bartorelli

36 Didattica d'artista in tv: Costruire è facile di Bruno Munari

Martina Rossi

44 Dalla formazione in scenografia alla Rai. Giosetta Fioroni costumista e Pino Pascali scenografo per la nascente televisione italiana

Anna Barenghi

58 «Noi non riprendiamo la realtà: la inventiamo»: Eugenio Carmi

Marco Maria Gazzano

70 L'invenzione della tv in Italia nell' esperienza di Mario Sasso
Paola Lagonigro

80 Obladì Obladà e Immagina: sperimentazioni tra video e computer art in tv

Pasquale Fameli

92 Se la tv è un white cube. Trasmissioni dell'arte nella televisione italiana degli anni Novanta

Damiano Garofalo

102 Note sul rapporto tra artisti e televisione italiana delle origini

Silvia Moretti

108 Gianni Toti. Occorrenze minimali per la Videopoesia Pietro Galifi

116 Grafica per una trasmissione tv di fine millennio: Altair4 Multimedia e Mediamente

|IIIIIIIIIIIIIIIIIIIIIII

Massimo Palma

124 La colpa in gioco. Elementi del Trauerspiel (a partire da Benjamin)

Alessio Bergamo

144 Rincorrere per non acchiappare. Un viaggio nell'esistenza di Wile E. Coyote e Road Runner, performer

Piersandra Di Matteo

174 Il piede e la sincope. Note su Edipo sveglia il tempo di Chiara Guidi 


\section{ABSTRACT}

Giosetta Fioroni e Pino Pascali hanno lavorato in anni differenti per la nascente televisione nazionale. La prima come costumista tra il 1955 e il 1957; mentre il secondo come scenografo tra il 1963 al 1967.

Questi lavori su commissione si situano per entrambi tra la formazione in scenografia all'Accademia di Belle Arti di Roma e il loro debutto come artisti di ricerca.

I programmi presi in esame, Gli interessi creati e Biblioteca di Studio Uno, rappresentano dei casi studio concreti del modo di operare di Fioroni e Pascali con il linguaggio prima scenico e poi televisivo, sebbene siano esperienze trascurate dalla letteratura dedicata ai due artisti.

Il contributo intende affrontare i lavori su commissione di Giosetta Fioroni e Pino Pascali per la Rai facendo emergere come questi episodi siano esemplari per comprendere come venga elaborata la formazione accademica in una fase intermedia del loro percorso artistico, fino a giungere alla sintesi compiuta nelle opere mature dei due artisti.

Giosetta Fioroni and Pino Pascali have worked in different years for the nascent national television. Fioroni was engaged as a costume designer between 1955 and 1957; while Pascali as set designer between 1963-1967.

For both - educated in scenography at the Academy of Fine Arts in Rome - these commissioned works precede their debut as research artists.

The programs examined, Gli interessi creati and Biblioteca di Studio Uno, represent case studies of Fioroni and Pascali's way of working with the scenic and then with the television language.

This contribution intends to examine the works commissioned by Rai to Giosetta Fioroni and Pino Pascali, highlighting how these episodes are exemplary to understand the impact of the academic training from the intermediate stage of their artistic career, up to the synthesis made in the mature works of the two artists.

\section{Dalla formazione in scenografia} alla Rai. Giosetta Fioroni costumista

e Pino Pascali scenografo per la nascente televisione italiana Martina Rossi

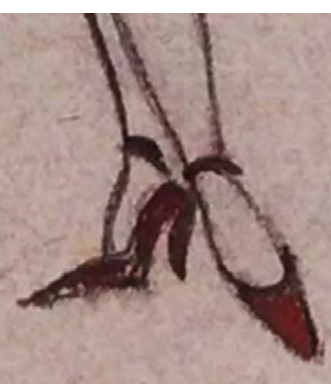

\section{Premessa}

Giosetta Fioroni e Pino Pascali condividono nel corso degli anni Cinquanta il medesimo percorso formativo in scenografia presso l'Accademia di Belle Arti di Roma. La prima e diretta applicazione di una tale impronta culturale è visibile però non in pièce teatrali, ma in alcuni programmi dell'allora nascente televisione italiana. Le esperienze lavorative di Fioroni e Pascali per la Rai, come costumista e scenografo, convivono con il loro debutto come artisti di ricerca segnando, parallelamente alle esposizioni ufficiali, i loro rispettivi esordi.

Nonostante l'ampia letteratura dedicata ai due protagonisti della neoavanguardia, quelle per la tv sono esperienze pressoché sconosciute sul fronte degli studi su Giosetta Fioroni, e scarsamente note sul fronte di Pino Pascali, se paragonate alla voluminosa bibliografia dedicata alla sua attività pubblicitaria. I lavori compiuti per la televisione sono per entrambi nettamente separati dal percorso intrapreso nell'arte di ricerca. D'altra parte, non possono essere sottovalutati superficialmente come meri risultati di incarichi su commissione. Difatti queste esperienze sono strettamente legate alla formazione dei due artisti e al contesto romano nel quale portarono avanti i loro studi. Inediti bozzetti di Fioroni e Pascali per alcuni sceneggiati televisivi italiani, rintracciati negli archivi perso- 
nali degli artisti ${ }^{1}$, ci dicono di più se relazionati agli anni di passaggio che vedono i due muoversi dalla formazione ricevuta sul fronte teatrale e scenotecnico in direzione degli esordi negli ambienti artistici più sperimentali.

Entrambi prendono parte a uno specifico genere di programma televisivo mosso da propositi didascalici e divulgativi, lo sceneggiato: riduzioni di celebri opere letterarie e, meno frequentemente, di sceneggiature inedite ${ }^{2}$. A partire già dal $1954^{3}$ il teleromanzo acquista un progressivo successo, accompagnato parallelamente da accese critiche su l'incolmabile lontananza del prodotto televisivo dal suo originale narrativo; sull'eliminazione di elementi potenzialmente "disturbanti" per il pubblico; e sul taglio fumettistico dell'intera costruzione drammaturgica e visiva ${ }^{4}$. I lavori di Fioroni e Pascali per la Rai si vanno a inscrivere in un momento aurale del loro percorso artistico di ricerca, come in una fase iniziatica per la stessa produzione televisiva, segnata, come è noto, da una vis maieutica concentrata su una alfabetizzazione anche letteraria degli spettatori ${ }^{5}$. In un testo del 1965 dedicato alla nuova tipologia drammaturgica televisiva è proprio il responsabile del palinsesto Rai di allora ${ }^{6}$, Sergio Pugliese, a prendere parola contro i preconcetti per i quali la tv sia il nemico della cultura e passatempo per uomini senza istruzione. Scrive Pugliese, il problema è più complesso e la televisione non è che il più appariscente dei fenomeni di una crisi di civilizzazione: il segno grafico non è più sufficiente a diffondere la conoscenza dei fatti, la scrittura si è fatta troppo lenta per una massa in continuo aumento che pretende di conoscere e valutare ${ }^{7}$. Una civiltà delle immagini la chiama Pugliese $^{8}$, adottando una definizione ricorrente del dibattito coevo ${ }^{9}$.

All'interno di questa nuova "realtà dell'immagine", i due giovani artisti devono però misurarsi con un linguaggio ancora alla ricerca di una propria specificità. Negli sceneggiati dei primi anni è evidente come l'alfabeto televisivo si mutui sui dettami scenotecnici teatrali, ridefinendosi sulle inedite esigenze dettate dallo studio televisivo e sulle nuove modalità di fruizione ${ }^{10}$.

1 Intendo ringraziare per la generosità e il supporto nella ricerca Giosetta Fioroni e l'archivio della Fondazione Goffredo Parise e Giosetta Fioroni, Roma, nelle persone di Elettra Bottazzi e Marta Bandini; inoltre l'archivio della Fondazione Pino Pascali, Polignano a Mare, Bari, nella persona di Antonio Frugis. Inoltre, intendo ringraziare Francesca Gallo per i suoi suggerimenti e Elisa Genovesi e Claudio Zambianchi per l'attenta lettura.

2 Cfr. A. Grasso, Enciclopedia della televisione, Garzanti, Milano 2008, p. 718

3 Cfr. I. Piazzoni, Storia delle televisioni in Italia. Dagli esordi alla web tv, Carocci editore, Roma 2020, p. 36; A.J. Di Santantonio, M. Beatrice Gallo, Storia della scenografia televisiva. Gli sceneggiati della Rai, vol. 1, Harman Publishing, London 2008, p. 9

4 Cfr. I. Piazzoni, Storia delle televisioni in Italia, cit., pp. 36-37.

5 Cfr. F. Monteleone, Storia della radio e della televisione in Italia. Costume, società e politica, Marsilio editori, Venezia, 1999, pp. 305-307; I. Piazzoni, Storia delle televisioni in Italia, cit., pp. 33-38.

6 Cfr. S. Pugliese, a cura di, Drammaturgia nuova. Raccolta di drammi e commedie scritte per la televisione, I Classici del teatro, vol. X, ERI-Edizioni Rai, Torino 1965.

7 Ivi, p. XVI.

$8 \mathrm{IVi}, \mathrm{p} . \mathrm{IX}$.

9 Si pensi al caso esemplare del numero La civiltà delle immagini, a cura di S. Morando, in «L'Almanacco Letterario Bompiano», 1963.

10 Cfr. A. Bellotto, La scenografia televisiva tra convergenze, divergenze ed autonomie, in G. Bettetini (a cura di), Forme scenografiche della televisione, Franco Angeli Editore, Milano 1982, pp. 29-33; A.J. Di Santantonio, M. Beatrice Gallo, Storia della scenografia televisiva, cit., p. 9.
I bozzetti e gli studi su commissione che i due artisti realizzano per questo nuovo mezzo linguistico assumono un ruolo rilevante all'interno della loro biografia, benché sia necessario sottolineare come questi lavori grafici siano per entrambi il risultato di esperienze circoscritte e secondarie rispetto alla ricerca da loro condotta sul medium pittorico e scultoreo. Tuttavia, i progetti per la Rai assumono un valore esemplare nel raccontarci il momento di passaggio tra la formazione in scenografia di Fioroni e Pascali - sotto l'impronta sperimentale di Toti Scialoja e quella più tradizionalista di Peppino Piccolo - e il loro riconoscimento ufficiale nell'alveo della nuova generazione romana. I disegni preparatori per i programmi tv, inoltre, sono le prove tangibili di come gli artisti utilizzino una siffatta istruzione scenica nella sfera pratica dello studio televisivo.

\section{Giosetta Fioroni costumista per gli sceneggiati di Guglielmo Morandi}

Giosetta Fioroni è la prima ad arrivare in Rai. Ancora molto giovane, a soli ventitré anni, partecipa come costumista a uno sceneggiato andato in onda nell'aprile del 1955 del regista Guglielmo Morandi. II teleromanzo intitolato Gli interessi creati11 è una riduzione della commedia di Jacinto Benavente (Los intereses creados, 1907) ispirata al teatro delle maschere. L'artista conserva nel suo archivio gli studi dedicati a questo lavoro: diciassette tavole con accurati progetti di costumi destinati a ogni singolo personaggio, bozzetti grafici a matita e tempera ai quali spesso vengono associati dei campioni di stoffa ${ }^{12}$. In questi disegni l'artista annota sia il nome del personaggio sia quello dell'attrice o dell'attore a cui il ruolo è affidato. Proprio attraverso queste indicazioni è stato possibile risalire al nome del programma del 1955 negli archivi delle Teche Rai. Finora, difatti, esigue sono state le informazioni sull'esperienza televisiva di Fioroni. L'artista ne parla in uno scritto autobiografico del $1990^{13}$, quando raccontando della stagione romana alla metà degli anni Cinquanta ricorda: «avevo brevemente lavorato come costumista per l'allora nascente televisione italiana con registi come Danza [N.d.A. Daniele D'Anza] e Morandi... Ma avevo lasciato anche quella attività [...]»14.

11 Gli interessi creati, di Giacinto Benavente, regia di Guglielmo Morandi, programma nazionale 22-24 aprile 1955

12 Nell'archivio della Fondazione Goffredo Parise e Giosetta Fioroni, Roma, sono conservati i seguenti bozzetti, 17 fogli sciolti, relativi ai personaggi: Leandro: Antonio Pierfederici; Leandro alla festa: Antonio Pierfederici; Donna Sirena: Elsa Vazzoler; Colombina: Luisa Baseggio; Risella: Cristina Fanton (il nome di Elsa Giliberti è cancellato e sostituito con Fanton); L'oste: Vinicio Sofia; La moglie di Pulcinella: Clara Crispo; Lo stalliere: ?; Silvia: Franca Maresa; Il segretario:?; Arlecchino: Gianni Bonagura; Laura: Donatella Gemmò; II Dottore Balanzone: Annibale Pirecli (il nome di Italo Pirani è cancellato e sostituito con Pirecli); Crispino: Marcello Moretti; ॥ capitano: Giuseppe Porelli. Senza indicazione degli interpreti: finto Zoppo; II segretario; Lo sguattero ('57 Cobelli).

13 G. Fioroni, Nota biografica per l'amico studioso d'Arte Mario Queseda che doveva trarne una biografia (inedito), 1990, in G. Bianchino (a cura di), Giosetta Fioroni, catalogo della mostra, Skira, Ginevra 2004, pp. 195-197 (p.196). Se ne riporta notizia anche in F. Di Castro (a cura di), Giosetta Fioroni. Opere su carta 1960-1990, catalogo della mostra, Elettra, Milano 1990, p. 163.

14 G. Fioroni, Nota biografica, cit., p. 196. 


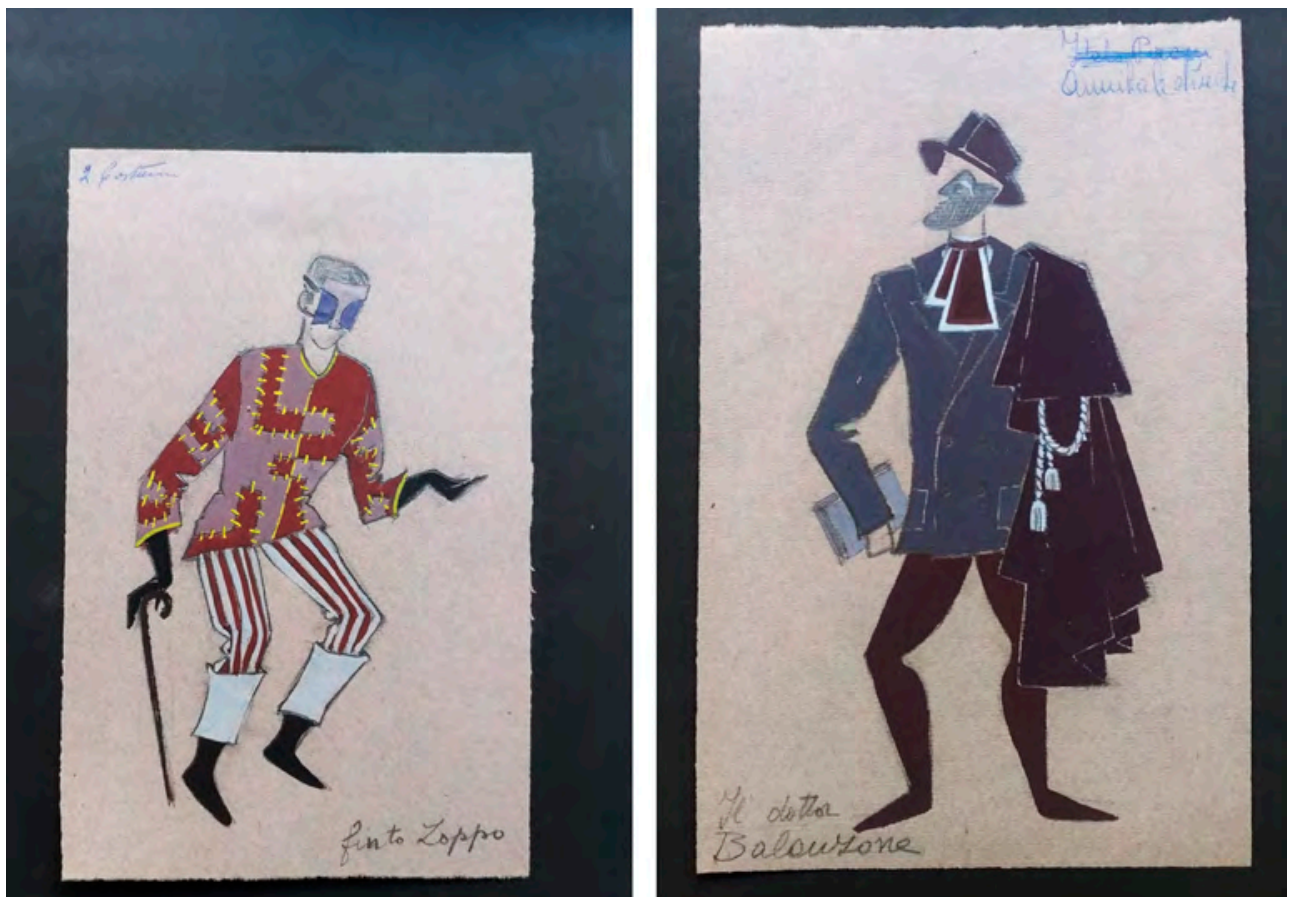

Giosetta Fioroni, studio dei costumi per "Finto zoppo" e "Il dottor Balanzone", Gli Interessi Creati, 1955. Courtesy of Fondazione Goffredo Parise e Giosetta Fioroni, Roma.

L'individuazione del primo programma nel quale si attesta la presenza dell'artista, non esaurisce però lo studio sui numerosi bozzetti non ancora identificati nel suo archivio personale. L'esperienza seppur breve presso la Rai non si limita alle messe in onda del regista Morandi. Lo attestano sia le dichiarazioni dell'artista sia alcuni studi che non rientrano all'interno de Gli Interessi creati ${ }^{15}$. Ne è un chiaro esempio il bozzetto per il personaggio dello "Sguattero" datato 1957, dove Fioroni annota "Gobetti", probabilmente il riferimento è al celebre attore Giancarlo. La mancanza di ulteriori dettagli non permette però una identificazione certa degli altri sceneggiati ai quali Giosetta Fioroni prende parte. A tutt'oggi si può in ogni modo affermare che il suo lavoro in Rai si avvia nel 1955 per terminare nel 1957. La collaborazione della giovane artista presso la nuova televisione italiana inizia, da quanto da lei riportato, proprio per il tramite diretto di Morandi ${ }^{16}$, uno dei pionieri della televisione ${ }^{17}$. I rapporti fra i due risalgono a un periodo precedente la messa in scena del 1955, ed è lo stesso regista a coinvolgere in prima persona l'artista affidandole il ruolo da costumista. Inizia in questo modo la prima esperienza sulla scena di Fioroni, così raccontata a Hans Ulrich Obrist: «Avevo circa vent'anni, e i registi erano cattivissimi, molto molto duri, tanto che io mi rifugiava spesso in bagno a piangere. Ero molto timida all'epoca. Feci i costumi per le riviste che si facevano allora [...]»18.

15 Per la lista dei bozzetti senza indicazione degli interpreti si veda nota n. 12.

16 Intervista a Giosetta Fioroni, di Marta Bandini, Elisa Genovesi e Martina Rossi. Roma, 9. VII.2020. 17 Cfr. A. Grasso, Enciclopedia della televisione, cit., p. 507.

18 G. Fioroni, H. U. Obrist, A Woman, Alone, Who Gets Bored, in C. Costa, F. Vezzoli (a cura di), TV
Il lavoro per Morandi si situa nella biografia dell'artista significativamente fra la sua frequentazione, seppur non assidua, dei corsi presso l'istituzione romana - a partire dall'ottobre 1950 - alla sua prima partecipazione a una esposizione istituzionale - la VII Quadriennale di Roma, nell'aprile 1956 - momento a partire dal quale la giovane studentessa deciderà di dedicarsi totalmente alla pittura e a cui farà seguito il suo trasferimento a Parigi alla fine del $1958^{19}$
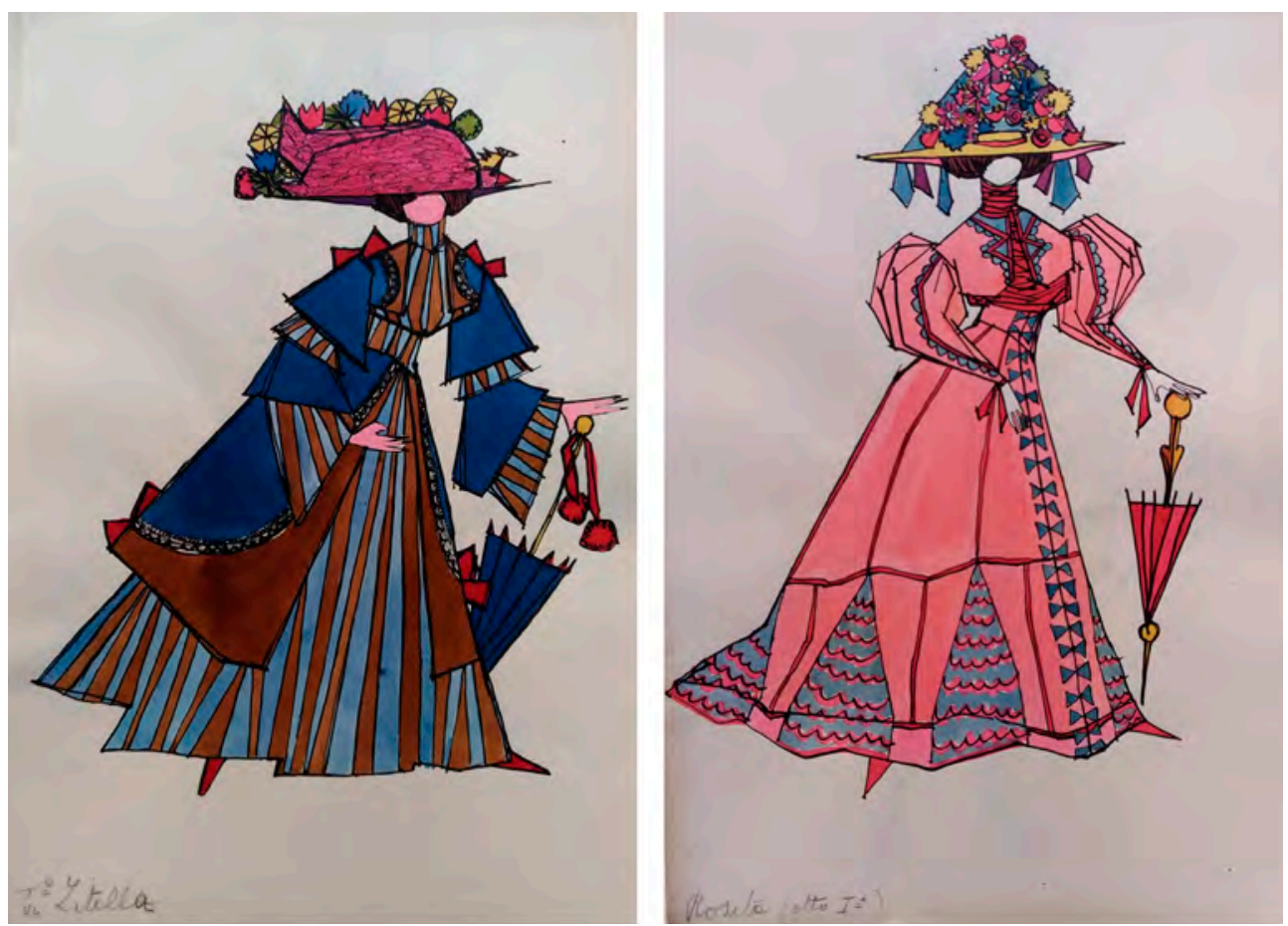

Thisitela

Giosetta Fioroni, studio dei costumi "IV Zitella" e "Rosita" per i corsi all'Accadem

L'esperienza di Giosetta Fioroni per la tv è però strettamente legata alla sua formazione in Accademia. Gli studi per Gli Interessi creati sono accompagnati da numerosi altri bozzetti di costumi realizzati dall'artista a partire dal 1950. Nelle tavole si alternano diverse soluzioni per i protagonisti delle opere teatrali che le vengono affidate come esercitazione in Accademia, mentre altri disegni sono dedicati allo studio filologico dei costumi d'epoca, in particolare il Seicento francese, tedesco e inglese ${ }^{20}$. La scrupolosità, I'abilità

70-Francesco Vezzoli guarda la Rai, catalogo della mostra, Fondazione Prada, Fondazione Prada 2017, pp. 147-164. Intervista pubblicata anche in H.U. Obrist, Giosetta Fioroni, in "Flash Art", 8 giugno 2017, https://flash---art.it/article/giosetta-fioroni/ (ultima consultazione 15.IX.2020).

19 Cfr. G. Celant (a cura di), Giosetta Fioroni, Skira, Ginevra-Milano 2009; e M. Meneguzzo, P. Mascitti, E. Bottazzi (a cura di), Giosetta Fioroni. Roma anni '60, catalogo della mostra, Silvana Editore, Cinisello Balsamo Milano 2016.

20 Due tavole di questo corpus di studi di costumi sono state già pubblicate in C. Gilman, Giosetta 
grafica e cromatica di questi bozzetti rivelano come Giosetta Fioroni si avvicini agli studi di scenografia con l'intento di diventare una costumista. Come afferma la stessa artista ${ }^{21}$, l'interesse per questo specifico aspetto della messa in scena la induce a iscriversi al corso tenuto all'Accademia e perdura per tutti gli studi, trovando una prima espressione pratica nei teleromanzi di Morandi.
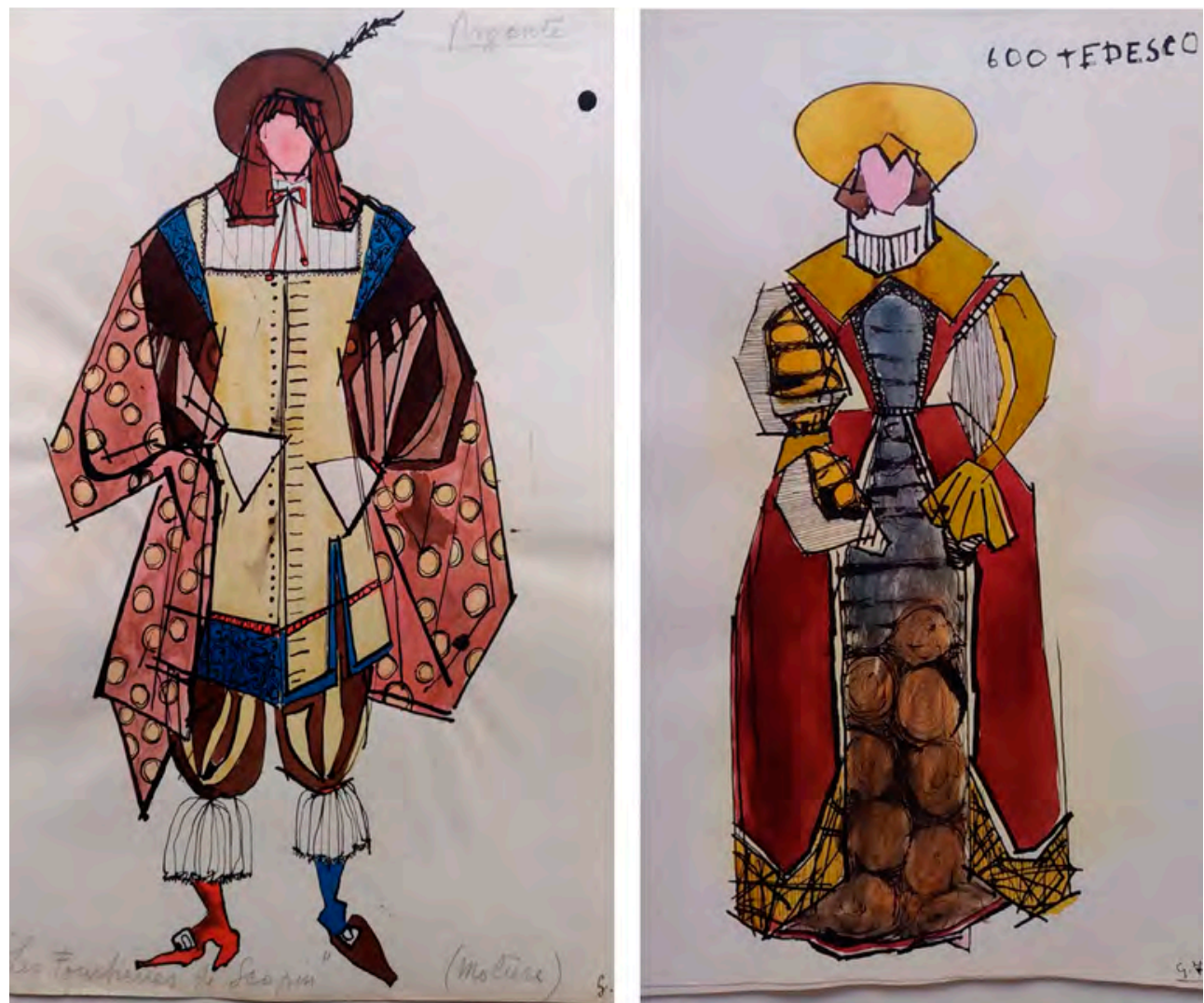

Giosetta Fioroni, studio di costumi per i corsi all'Accaden

L'artista ha ricordato in diverse sedi come sia stato fondativo per lei l'incontro dal 1953 con il suo insegnante di scenotecnica e storia del teatro, Toti Scialoja ${ }^{22}$. Rispetto al titolare del suo corso, Peppino Piccolo, l'artista aveva portato in Accademia l'aggiornamento sul fronte teatrale delle maggiori sperimentazioni d'avanguardia. Giosetta Fioroni ricorda

Fioroni: L'Argento, catalogo della mostra, The Drawing Center's, New York 2013, pp. 44-45 (pl. 20-21: Costume design from Rome's Accademia di Belle Arti di Roma, 1950).

21 Intervista a Giosetta Fioroni, cit.

22 Fra le numerose fonti bibliografiche si rimanda a G. De Marco, Piazza del popolo: 1950-1960, in Gli anni originali, «La Tartaruga. Quaderni d'arte e letteratura», v.S., marzo 1989, n. 5-6, pp. 105-128 (p. 109). come solo con Scialoja potesse parlare di teatro o di costumi senza negare anche l'interesse per la pittura23. Nella prima metà degli anni Cinquanta, e ne sono una prova gli studi qui esaminati, Fioroni cerca di conciliare il lavoro sui vestiti di scena con la nuova aspirazione di diventare artista. Con il suo maestro poteva accedere a una concezione interdisciplinare del fare artistico, per la quale realizzare i costumi non è più solo lavoro di mestiere ma parte integrante della mise en scène. Da questo punto di vista, le teorizzazioni di Scialoja ${ }^{24}$ trovano seguito nella sua allieva Fioroni. Ne è chiara eco la Carmen per la regia di Alberto Arbasino del 196725, dove l'artista, questa volta a teatro, ritorna a progettare i vestiti di scena. Fra le strutture di Vittorio Gregotti l'artista realizza costumi con forme e colori che «alludevano con simbologie semplificate ai temi della pittura contemporanea. I pois nelle multiversioni Pop e i segni, le strisce, i gonfiori, gli ornamenti e i trucchi... in una costante stilizzazione» ${ }^{26}$. Al Teatro Comunale di Bologna l'immaginario visivo di Fioroni assume le forme di costumi che conquistano lo spazio con il proprio ingombro entrando in relazione con l'architettura delle scene.

Dalla sua formazione, alla sua esperienza in Rai, fino alle sperimentazioni al Teatro Comunale di Bologna, in Giosetta Fioroni rimane costante l'interesse verso i vestiti di scena intesi, nell'ottica dell'insegnamento di Scialoja, come "accenti pittorici" in movimento ${ }^{27}$

\section{Pino Pascali scenografo di Biblioteca di Studio Uno}

Poco meno di dieci anni dopo, anche Pino Pascali compie un simile percorso che lo vede dall'Accademia giungere in Rai come scenografo. Rispetto all'ampia bibliografia dedicata alla sua attività nella pubblicità e nella realizzazione di sigle televisive, non altrettanta attenzione è stata posta sui progetti scenografici dell'artista ${ }^{28}$. Pascali terminato il percorso di studi nel 1959, ottiene l'incarico come aiuto scenografo di Carlo Cesarini da Senigallia per i programmi Studio Uno (1961-1966), Napoli contro tutti (1964), Biblioteca di Studio Uno (1964) e Scala Reale (1966-1967). La collaborazione proseguirà per circa quattro anni, dal 1963 al 1967, anno nel quale gli viene offerto l'incarico di responsabile delle scenogra-

23 Intervista a Giosetta Fioroni, cit.

24 Cfr. T. Scialoja, Premesse per una moderna scenografia, in «Mercurio», n. 3, a. I, novembre 1944, pp. 142-148; T. Scialoja, Danza-Pittura, in «Mercurio», n. 17, a. III, gennaio 1946, pp. 143-151. Ripubblicati in A. Tarasco (a cura di), Toti Scialoja critico d'arte. Scritti in "Mercurio", 1944-1948, Gangemi editore, Roma 2015, pp. 13-19, 16-125.

25 Carmen, musiche di G. Bizet, direttore: P. Dervaux, regia: A. Arbasino, coreografie: C. Faraboni, scene: V. Gregotti, costumi: G. Fioroni, Bologna, Teatro Comunale, 31 gennaio, 2 e 5 febbraio 1967.

26 G. Fioroni, La nostra Carmen, in Quella Carmen. Alberto Arbasino, Giosetta Fioroni, Vittorio Gregotti, catalogo della mostra, Galleria dell'Oca, Roma s.d. [1989].

27 Cfr. T. Scialoja, Premesse per una moderna scenografia, cit., p. 54

28 Sulle scenografie di P Pascali per la Rai vedi: L. Cherubini, Pascali e la tv: l'originale esperienza di scenografo per il programma "Biblioteca di studio 1" del Quartetto Cetra, in "Quadri\&sculture», n. 27, maggio 1997, p. 66; M. Giusti, filmografia, in D. Ferraria (a cura di), Pascali, Disegni per la pubblicità, catalogo della mostra, Emme Otto, Roma 2008, pp. 61-63; C. Lodolo, 32 anni di vita circa. Pino Pascali raccontato da amici e collaboratori, Carlo Cambi editore, Poggibonsi (SI) 2012. 
fie di Partitissima lavoro che rifiuterà per dedicarsi totalmente all'arte di ricerca ${ }^{29}$. Difficile spesso determinare, sia nei lavori pubblicitari sia nelle scene, l'effettivo apporto autoriale di Pascali ${ }^{30}$. Tuttavia, per quanto riguarda le scenografie, vengono in soccorso ventitré accurati progetti grafici realizzati dall'artista per Biblioteca di Studio Uno, rintracciati nel suo archivio personale ${ }^{31}$

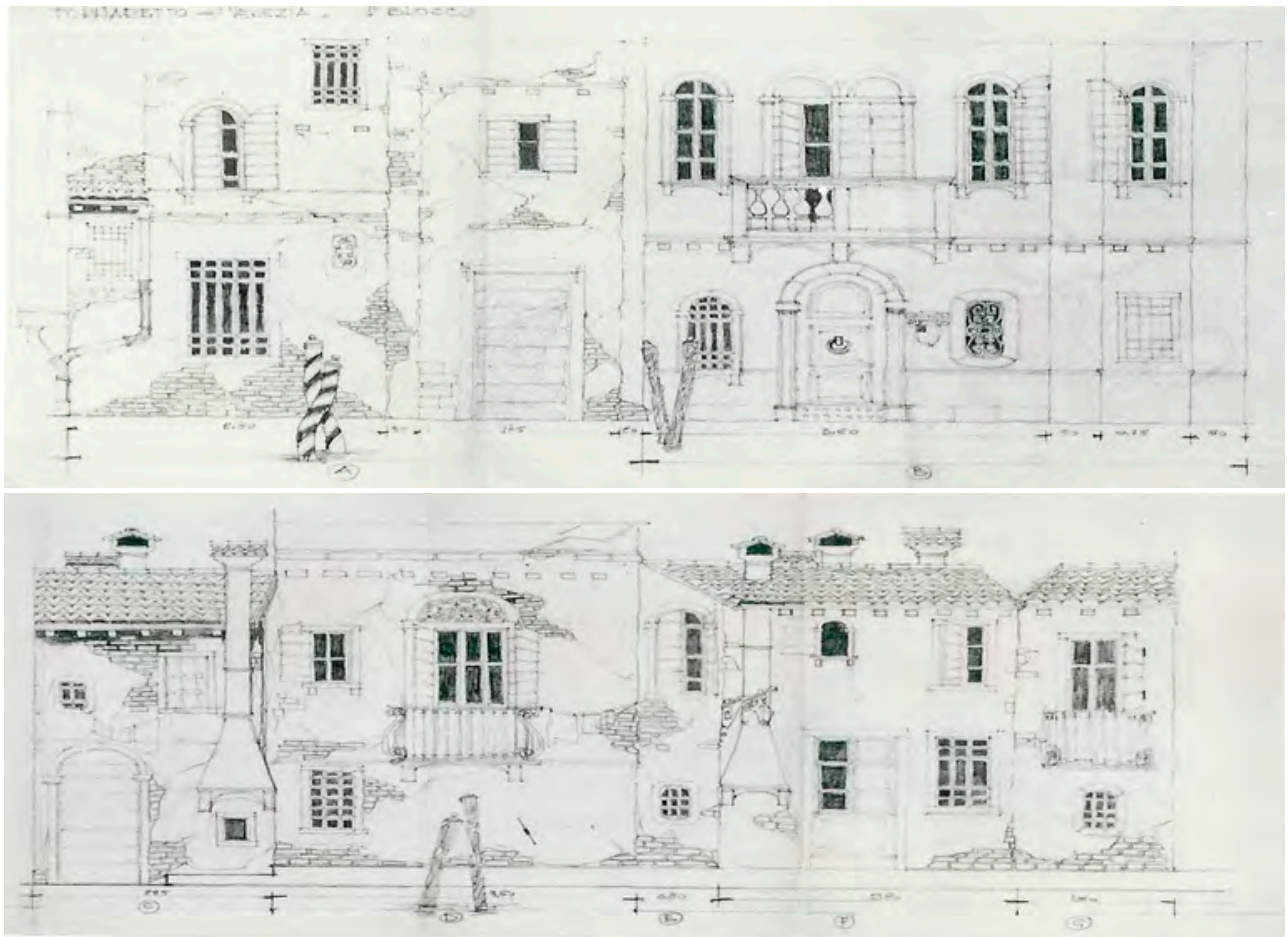

Pino Pascali, bozzetto scenografico, Il Fornaretto di Venezia, Biblioteca di Studio Uno, 1964. Courtesy Fondazione Pino Pascali. Archivio Pino Pascali, Polignano a Mare, Bari.

29 Ricordando Pino Pascali, fogli singoli non rilegati, manoscritti, s.d. [1969], Faldone Pino Pascali (1935-1959), 21/5, Roma, Galleria Nazionale d'Arte Moderna, archivio bioiconografico.

30 Ibidem

31 I bozzetti scenografici di Pino Pascali per Biblioteca di Studio Uno sono: L'Odissea, II fornaretto di Venezia; II Conte di Montecristo; La Storia di Rossella O'Hara (da Via col vento); La primula rossa: I tre moschettieri; Al Grand Hotel; I promessi sposi, Polignano a Mare (BA), archivio della Fondazione Museo Pino Pascali.
Lo sceneggiato musicale va in onda dal febbraio all'aprile nel 1964, la regia è affidata ad Antonello Falqui e gli autori sono gli stessi artefici di Studio Uno ${ }^{32}$. II varietà è considerato uno delle prime grandi produzioni della televisione italiana: otto puntate, ognuna delle quali incentrata su un'opera letteraria. Partendo dalla sigla si comprende chiaramente l'intento con il quale nasce la trasmissione, quello di far approcciare un vasto pubblico alla letteratura. Nella sigla i ballerini danzano e cantano all'interno di una biblioteca invitando lo spettatore in un viaggio nel mondo della narrativa. Per tutte le puntate si adotta un linguaggio semplificato ricorrendo alla costante presenza di un cantastorie, mentre la quasi totalità dei monologhi e dei dialoghi non vengono recitati, ma cantati dagli attori, riscritti su musica leggera dal Quartetto Cetra. II risultato è un teleromanzo fra parodia e musica ${ }^{\beta 3}$

Di questa breve parentesi di Pascali presso la Rai parla una lettera scritta dai genitori appena dopo la sua scomparsa ${ }^{34}$. In quest'ultima si trova conferma di come l'artista considerasse la collaborazione con la televisione un lavoro legato alle necessità contingenti e parallelo al suo percorso di ricerca:

Pino attraversò un duro periodo d'inquietudine sacrificando la sua libertà accettò di collaborare nel quadro artistico della INCOM, indi nella direzione artistica della Saraceni Cinematografica Pubblicitaria in Roma, ed infine per quattro anni al Centro di Produzione Rai tv di via Teulada, quale aiuto scenografo dello Studio Uno 35

La collaborazione dell'artista per gli sceneggiati si pone, anche in questo caso, in un momento di passaggio: qualche anno dopo il diploma in scenografia conseguito all'Accademia nel 1959, e solo un anno prima del suo debutto come artista di ricerca presso la galleria La Tartaruga, all'alba del 1965.

Tuttavia, la collaborazione con Biblioteca sembra inscriversi, come visto pocanzi per Fioroni, all'interno delle esperienze formative di Pascali. Difatti, i numerosi studi grafici dell'artista, destinati allo sceneggiato, testimoniano le sue capacità disegnative, l'abilità nella progettazione delle scene e la familiarità con il mestiere da scenografo. La fedeltà con cui gli studi di Pascali vengono trasposti nelle ambientazioni in studio forniscono un'ulteriore prova di questa sua straordinaria perizia. Ne sono un esempio i bozzetti per Il Conte di Montecristo, dove una dettagliata nave ormeggiata al porto, la taverna, l'insegna con la sagoma di una balena sono realizzati meticolosamente negli studi di via Teulada. Lo stesso si può affermare per i disegni della prigione in cui è rinchiuso Edmond Dantès e la cella di Faria. In quest'ultimi studi, Pascali evidenzia graficamente anche il blocco di pietra che verrà spostato dal marinaio per far accedere alla sua cella l'abate, come anche i cunicoli utilizzati dagli attori durante le riprese.

La vicinanza di questa esperienza con la prima personale alla galleria di Plinio De Marti-

32 Biblioteca di Studio Uno, varietà-sceneggiato, otto puntate, 15 febbraio-18 aprile 1964, regia: A. Falqui, autori: A. Falqui, D. Verde, II Quartetto Cetra, orchestra: B. Canfora. Cfr. A. Grasso, Enciclopedia della televisione, cit., pp. 81-82, 143, 263. Come scenografo è spesso segnalato Cesarini da Senigallia, anche se il suo nome non viene riportato nei titoli di testa o di coda, Giuseppe Pascali è indicato come collaboratore alle scene nei titoli di coda.

33 Cfr. I. Piazzoni, Storia delle televisioni in Italia, cit., p. 91

34 Ricordando Pino Pascali, cit.

35 Ibidem. 
is non comporta invece una esplicita condivisione di soluzioni iconografiche tra i lavori su commissione e le opere esposte nel 1965. Un elemento di congiunzione può essere piuttosto rintracciato nella tecnica costruttiva, lo scheletro di centine lignee e tela, che Pascali sembra adottare sia negli sceneggiati sia nelle sculture presentate alla Tartaruga, direttamente estrapolata dalla sua formazione accademica.
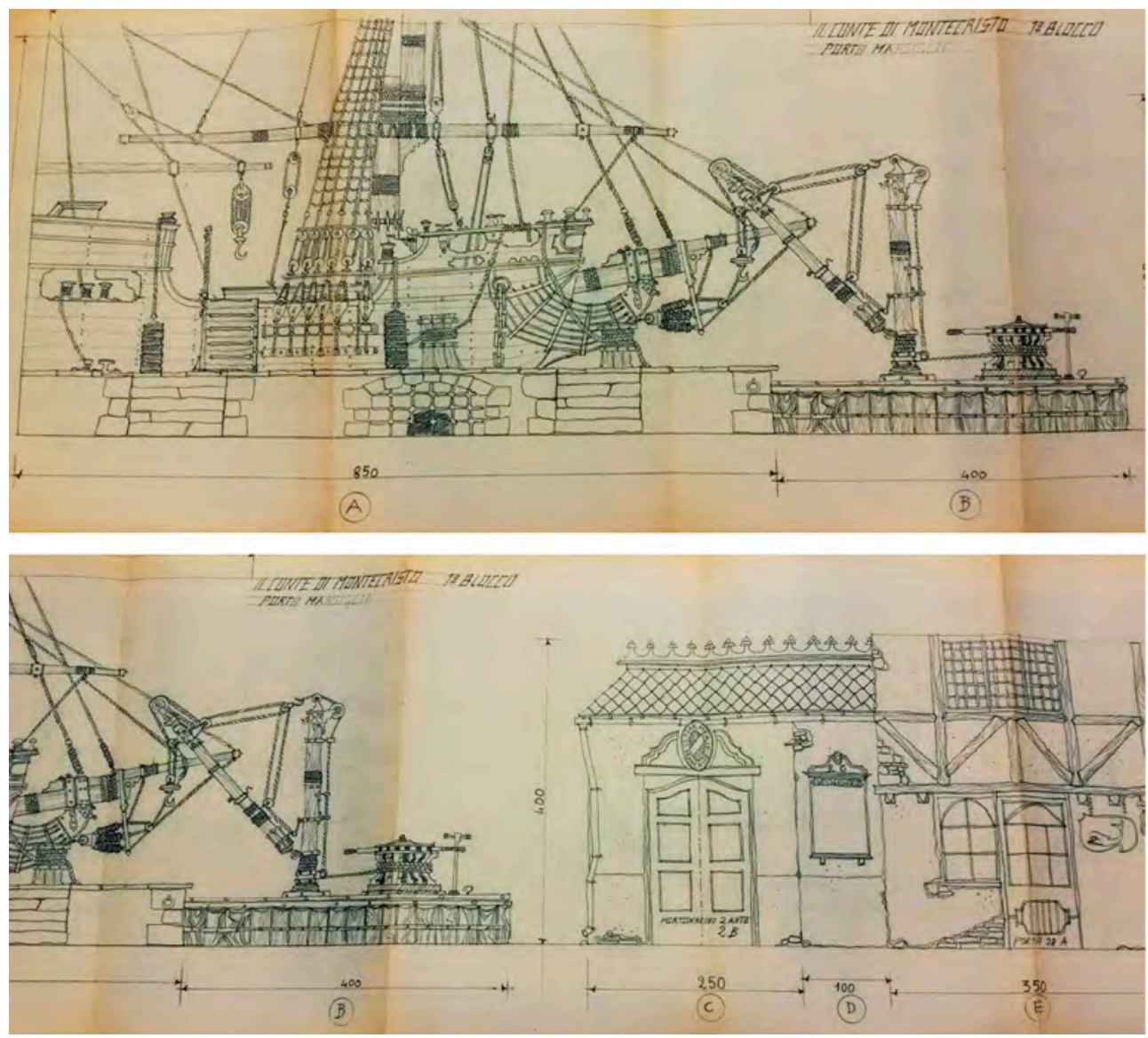

Pino Pascali, dettagli del bozzetto scenografico per il "porto di Marsiglia", Il Conte di Montecristo, Biblioteca di Studio Uno, 1964. Courtesy Fondazione Pino Pascali. Archivio Pino Pascali, Polignano a Mare, Bari.

Attraverso Biblioteca diventa evidente come la formazione dell'artista in campo scenico sia composta da differenti riferimenti culturali. Nelle scenografie per il teleromanzo si alternano ambientazioni canonicamente intese come accompagnamento didascalico al testo, a soluzioni più giocose e ironiche (si pensi alla comica rappresentazione dell'identikit di Mrs. Hyde o gli oggetti ingigantiti di uso comune nella caverna di Polifemo come l'enorme tazzina, un pettine con un capello spesso quanto una fune).

I lavori di Pascali per la Rai evidenziano come la sua formazione non si forgi soltanto sullo sperimentalismo di Toti Scialoja. Seppur rimanga fondamentale l'assidua frequentazione di Pascali dei corsi di scenotecnica del pittore e indiscutibili siano le ripercussioni nella sua opera matura; si deve altresì riconoscere come l'artista abbia attinto, soprattutto per i lavori in Rai, anche agli insegnamenti più tradizionali legati al fare tecnico del mestiere, affrontati probabilmente nel corso di scenografia di Peppino Piccolo.
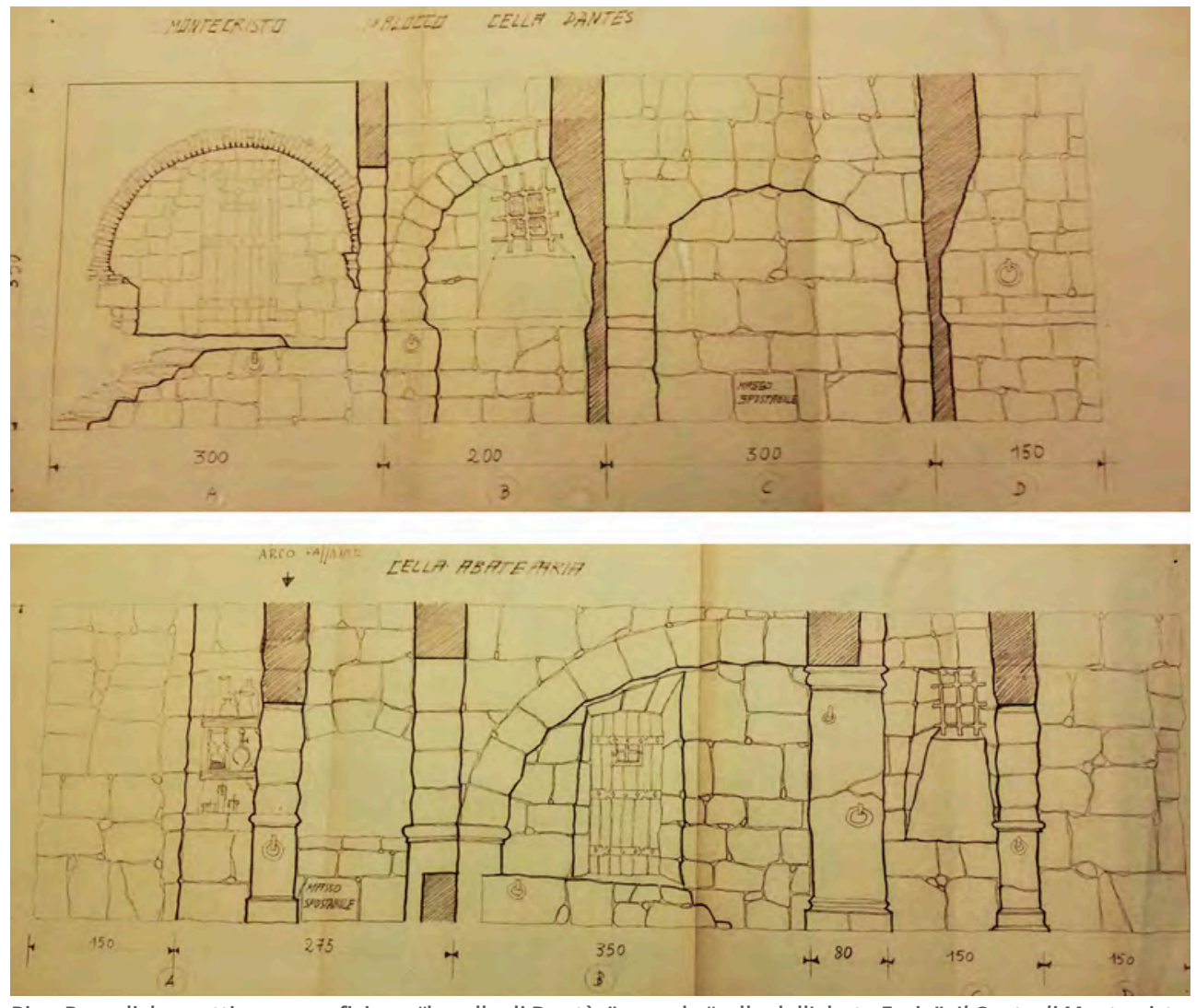

Pino Pascali, bozzetti scenografici per "la cella di Dantès" e per la "cella dell'abate Faria", Il Conte di Montecristo Biblioteca di Studio Uno, 1964. Courtesy Fondazione Pino Pascali. Archivio Pino Pascali, Polignano a Mare, Bari.

Nelle puntate dello sceneggiato del 1964, partendo dagli studi grafici a disposizione, si può cogliere questa non univocità dell'istruzione scenica di Pascali. Del resto per Piccolo l'artista aveva realizzato anche dei modellini e dei progetti grafici per le ipotetiche scenografie dell'Amleto, del Giulio Cesare di Shakespeare, Tristano e Isotta di Wagner e l'Arlecchinata di Salieri36. Studi presentati in occasione di una mostra di giovani scenografi appena diplomati dall'Accademia nel luglio 1959 al Palazzo Collicola di Spoleto, in occasione della seconda edizione del Festival dei Due Mondi.

Questa differenza intrinseca nella formazione culturale di Pascali - fra lavoro artigianale, immaginario colto e soluzioni visive sperimentali - viene colto nel primo testo dove si fa accenno al lavoro per la tv dello "scultore". In un articolo su «Metro» del 1968, Maurizio Fagiolo chiamato a ricordare due artisti scomparsi pochi mesi prima, Lucio Fontana e Pino Pascali, scrive: «Pascali vede tutto gigantesco, e tutto realizza coi mezzi del grande artigiano: ricordo le sue molte 
scenografie per la tv soprattutto nel 1965 (Studio Uno per esempio). Agli inizi, ammetto, era difficile afferrare il senso di questa ricerca tesa sempre a scavalcarsi e negare se stessa» ${ }^{37}$.

I lavori per la Rai sono un ulteriore tassello nel percorso compiuto da Pascali nello sviluppo della sua formazione come scenografo. Nella sua ricerca sperimentale l'artista troverà la sintesi fra le tecniche proprie del mestiere e la spinta colta e sperimentale dei suoi studi teatrali attraverso i quali la scultura-oggetto si fa ambiente e spazio per la messa in scena.

Gli studi grafici di Giosetta Fioroni e di Pino Pascali realizzati per gli sceneggiati tv ci permettono di addentrarci in una fase cruciale di passaggio per i due artisti e chiariscono l'importanza della loro formazione come costumista e scenografo, un'educazione scenica che troverà inedite soluzioni nelle loro ricerche mature. 